\title{
The Stability and Stabilization of Stochastic Delay-Time Systems
}

\author{
Gang Li ${ }^{1,2}$ and Ming Chen ${ }^{1}$ \\ ${ }^{1}$ School of Mathematics and System Science, Shandong University of Science and Technology, Qingdao 265590, China \\ ${ }^{2}$ College of Electrical Engineering and Automation, Shandong University of Science and Technology, Qingdao 265590, China \\ Correspondence should be addressed to Gang Li; ligangccm@163.com
}

Received 17 December 2013; Revised 17 April 2014; Accepted 1 May 2014; Published 3 June 2014

Academic Editor: Wuquan Li

Copyright (C) 2014 G. Li and M. Chen. This is an open access article distributed under the Creative Commons Attribution License, which permits unrestricted use, distribution, and reproduction in any medium, provided the original work is properly cited.

\begin{abstract}
The aim of this paper is to investigate the stability and the stabilizability of stochastic time-delay deference system. To do this, we use mainly two methods to give a list of the necessary and sufficient conditions for the stability and stabilizability of the stochastic time-delay deference system. One way is in term of the operator spectrum and $H$-representation; the other is by Lyapunov equation approach. In addition, we introduce the notion of unremovable spectrum of stochastic time-delay deference system, describe the $\mathrm{PBH}$ criterion of the unremovable spectrum of time-delay system, and investigate the relation between the unremovable spectrum and the stabilizability of stochastic time-delay deference system.
\end{abstract}

\section{Introduction}

The stochastic time-delay system is one of the fundamental research branches in the theory of control systems, which is usually applied in the fields of electronics, machinery, chemicals, life sciences, economics, and so on. As is well known, the stability is an essential concept in linear system theory, which is relative to the system matrix root-clustering in subregions of the complex plane, and also the spectral operator approach is effective in the study of the eigenvalue placement of a matrix (see [1]). Since two classic books [2, 3] appeared, stochastic stability and stabilization of Itô differential systems have been investigated by many researchers for several decades; we refer the reader to [4-6] and the references therein. More specifically, for linear time-invariant stochastic (LTIS) systems, most work is concentrated on the investigation of mean square stabilization, which has important applications in system analysis and design. Some necessary and sufficient conditions for the mean square stabilization of LTIS systems have been obtained in terms of generalized algebraic Riccati equation (GARE) or linear matrix inequality (LMI) in [7-14] or spectra of some operators in [5, 9, 15]. For the stochastic delay-time systems, the present results were mainly obtained by Lyapunov functional approach. We concentrate our attention upon the stability and stabilization of stochastic systems by the operator spectrum.
The structure of this paper is as follows. In Section 2, with the aid of the operator spectrum, $H$-representation, and Lyapunov equation approach, some necessary and sufficient conditions are given for the stability and the stabilizability of stochastic delay-time systems. In Section 3, the unremovable spectrum of stochastic delay-time systems is introduced, and $\mathrm{PBH}$ criterion of the stabilizability of stochastic delay-time systems is presented.

For convenience, we adopt the following traditional notations. $S^{n}$ : the set of all symmetric matrices, whose components may be complex; $N=\{0,1,2, \ldots\} ; A^{\prime}(\operatorname{Ker}(A))$ : the transpose (kernel space) of a matrix $A ; A \geq 0(A>0)$ is a positive semidefinite (positive-definite) symmetric matrix $A$; $I$ : identity matrix; $\sigma(L)$ : spectral set of the operator or matrix $L ; C^{-}\left(C^{-0}\right)$ : the open left (closed left) hand side complex plane. $D(0, \alpha)=\{\lambda \mid\|\lambda\|<\alpha\} ;\|\cdot\|$ is the $l_{2}$-norm; $L_{\mathscr{F}_{t}}^{2}\left(R^{+}, R^{n_{x}}\right)$ : space of nonanticipative stochastic processes $x(t) \in R^{n_{x}}$ with respect to an increasing $\sigma$-algebra $\left\{\mathscr{F}_{t}\right\}_{t \geq 0}$ satisfying $E\|x(t)\|^{2}<\infty$. Finally, we make the assumption throughout this paper that all systems have real coefficients.

\section{The Stability of Stochastic Delay-Time Systems}

In this section, we will investigate the stability and stabilizability of the stochastic time-delay deference system using 
the spectrum of operator and Lyapunov equation approach. At first, we introduce a Lyapunove operator. Consider the following linear difference system with constant delays:

$$
\begin{aligned}
x(t+1)= & F_{0} x(t)+G_{0} x(t) w(t) \\
& +\sum_{j=1}^{m}\left[F_{j} x(t-j)+G_{j} x(t-j) w(t)\right], \quad t \in N,
\end{aligned}
$$

with the initial condition

$$
x(k)=\varphi(k), \quad k=0,-1,-2, \ldots,-m .
$$

Here, $x \in R^{n}$ is a column vector, $F_{j}, G_{j} \in R^{n \times n}, j=0,1, \ldots, m$, are constant coefficient matrices, $\varphi(k) \in R^{n}$ is a deterministic initial condition, $\{w(t) \in R, t \in N\}$ is a sequence of real random variables defined on a complete probability space $\left\{\Omega, \mathscr{F}, \mathscr{F}_{t}, \mu\right\}$ which is a wide sense stationary, second-order process with $E(w(t))=0$ and $E(w(t) w(s))=\delta_{s, t}$, where $\delta_{s, t}$ is the Kronecker delta with $\mathscr{F}_{t}=\sigma\{w(s): 0 \leq s \leq t\}$.

Definition 1. The trivial stationary solution $x(t)=0$ of the system (1) is called mean square stable if, for any arbitrarily small number $\varepsilon>0$, there exists a number $\delta>0$, when $\|\varphi\|<$ $\delta$, such that

$$
E\|x(t)\|^{2}<\varepsilon
$$

for a solution $x(t)=x(t, \varphi)$ of $(1)$.

Definition 2. The trivial stationary solution $x(t)=0$ of the system (1) is called asymptotically mean square stable if it is stable in the sense of Definition 1 and, moreover, any solution $x(t)=x(t, \varphi)$ of $(1)$ satisfies

$$
\lim _{t \rightarrow+\infty} E\|x(t)\|^{2}=0
$$

We consider the problem of finding criteria and sufficient conditions for the mean square asymptotic stability of the trivial stationary solution $x(t)=0$ by operator spectra. Since the stochastic system (1) is a system with time-delays, it seems impossible to construct the operator directly for (1) like the operator in [15]. So, first of all, we introduce the following column vector $\widetilde{x}(t)$ of new variables of dimension $n(m+1)$ :

$$
\tilde{x}(t)=\left[x^{\prime}(t), x^{\prime}(t-1), \ldots, x^{\prime}(t-m)\right]^{\prime} .
$$

The stochastic system (1) with time-delays can now be written in the form of an equivalent stochastic system of dimension $n(m+1)$ without delay; namely,

$$
\tilde{x}(t+1)=[F+G \omega(t)] \tilde{x}(t),
$$

where $F$ and $G$ denote the following $n(m+1) \times n(m+1)$ matrices:

$$
\begin{aligned}
F & =\left(\begin{array}{ccccc}
F_{0} & F_{1} & \cdots & F_{m-1} & F_{m} \\
I & 0 & \cdots & 0 & 0 \\
\vdots & \vdots & \vdots & \vdots & \vdots \\
0 & 0 & \cdots & I & 0
\end{array}\right), \\
G & =\left(\begin{array}{ccccc}
G_{0} & G_{1} & \cdots & G_{m-1} & G_{m} \\
0 & 0 & \cdots & 0 & 0 \\
\vdots & \vdots & \vdots & \vdots & \vdots \\
0 & 0 & \cdots & 0 & 0
\end{array}\right) .
\end{aligned}
$$

If we set $X(t)=E \tilde{x}(t) \tilde{x}^{\prime}(t), X(t)$ satisfies the following difference equation:

$$
X(t+1)=F X(t) F^{\prime}+G X(t) G^{\prime} .
$$

Motivated by (8), we introduce the following linear Lyapunov operator:

$$
\mathscr{L}_{F, G}: X \in S^{n(m+1)} \longmapsto F X(t) F^{\prime}+G X(t) G^{\prime} \in S^{n(m+1)} .
$$

With the use of the Kronecker matrix product, the matrix equation (8) can be rewritten in the vector matrix form as follows:

$$
\vec{X}(t+1)=\widehat{F} \vec{X}
$$

where $\vec{X}(t)$ denotes the $n^{2}(m+1)^{2}$-dimensional column vector

$$
\begin{aligned}
\vec{X}(t)= & {\left[X_{1,1}(t), \ldots, X_{1, n}(t), \ldots,\right.} \\
& \left.X_{1, n(m+1)}(t), \ldots, X_{n(m+1), n(m+1)}(t)\right]^{\prime}
\end{aligned}
$$

and $\widehat{F} \in R^{n^{2}(m+1)^{2} \times n^{2}(m+1)^{2}}$ has the form $\widehat{F}=F \otimes F+G \otimes G$.

Now, we are in a position to give a spectral description for the stability of system (1) by $H$-representation in [14].

Lemma 3. Let $H_{n(m+1)}$ be a $n^{2}(m+1)^{2} \times(n(m+1)[n(m+1)+$ $1] / 2)$ matrix and $\operatorname{rank}\left(H_{n(m+1)}\right)=(n(m+1)[n(m+1)+1]) / 2$; then $H_{n(m+1)}^{\prime} H_{n(m+1)}$ is invertible.

Theorem 4. The trivial solution $x(t)=0$ of system (1) is asymptotically mean square stable if and only if $\sigma\left(\mathscr{L}_{F, G}\right) \subset$ $D(0,1)$.

Proof. If we set $X(t)=E \tilde{x}(t) \tilde{x}^{\prime}(t), X(t)$ satisfies

$$
\begin{gathered}
X(t+1)=F X(t) F^{\prime}+G X(t) G^{\prime}, \\
X(k)=\tilde{x}(k) \tilde{x}^{\prime}(k) \in S^{n(m+1)}, \\
k=0,-1,-2, \ldots,-m, \quad t \in N .
\end{gathered}
$$

Since $X(\cdot)$ is real symmetric, (12) is a linear matrix equation with $n(m+1)[n(m+1)+1] / 2$ different variables; that is, it is in fact an $n(m+1)[n(m+1)+1] / 2$ th-order linear system. We define a map $\widetilde{\mathscr{L}}$ from $S^{n(m+1)}$ to $C^{n(m+1)[n(m+1)+1] / 2}$ as follows. 


$$
\begin{aligned}
& \text { For any } Y=\left(Y_{i j}\right)_{n(m+1) \times n(m+1)} \in S^{n(m+1)} \text {, set } \\
& \begin{aligned}
\widetilde{Y}=\widetilde{\mathscr{L}}(Y)=( & Y_{11}, \ldots, Y_{1, n(m+1)}, \ldots, Y_{n(m+1)-1, n(m+1)-1}, \\
& \left.Y_{n(m+1)-1, n(m+1)}, \ldots, Y_{n(m+1), n(m+1)}\right)^{\prime} ;
\end{aligned}
\end{aligned}
$$

then there exists an unique matrix $\theta\left(H_{n(m+1)}\right) \quad \epsilon$ $R^{(n(m+1)[n(m+1)+1] / 2) \times(n(m+1)[n(m+1)+1] / 2)}$, by $H$-representation of [14], such that (12) is equivalent to

$$
\begin{gathered}
\widetilde{X}(t+1)=\widetilde{\mathscr{L}}\left(\mathscr{L}_{F, G}(X)\right)=\theta\left(H_{n(m+1)}\right) \widetilde{X}(t), \\
\widetilde{X}(k)=\left[H_{n(m+1)}^{\prime} H_{n(m+1)}\right]^{-1} H_{n(m+1)}^{\prime} \\
\times \vec{X}(k) \in R^{n(m+1)[n(m+1)+1] / 2}, \\
k=0,-1,-2, \ldots,-m, \quad t \in N,
\end{gathered}
$$

where $\theta\left(H_{n(m+1)}\right)=\left[H_{n(m+1)}^{\prime} H_{n(m+1)}\right]^{-1} H_{n(m+1)}^{\prime}[F \otimes F+$ $G \otimes G] H_{n(m+1)}, \widetilde{X} \in R^{n(m+1)[n(m+1)+1] / 2}$. Obviously, since the system of (14) for moments is deterministic, the proof of the theorem is carried out by the standard method for deterministic difference equations. Seeking the general solution of system (14) in the exponential form $\widetilde{X}(t)=c \lambda^{t}$, where $c, \lambda=$ const, we arrive at the characteristic equation $\operatorname{det}\left(\lambda I-\theta\left(H_{n(m+1)}\right)\right)=0$. That is, $\lim _{t \rightarrow+\infty} E \tilde{x}(t) \tilde{x}^{\prime}(t)=$ $\lim _{t \rightarrow+\infty} X(t)=0 \Leftrightarrow \lim _{t \rightarrow+\infty} \widetilde{X}(t)=0 \Leftrightarrow \sigma\left(\theta\left(H_{n(m+1)}\right)\right) \subset$ $D(0,1)$.

By (9) and (14), for any eigenvalue $\lambda$ and its corresponding eigenvector $Y=\left(Y_{i j}\right)_{n \times n} \in S^{n}$ of $\mathscr{L}_{F, G}$, from $\mathscr{L}_{F, G}(Y)=$ $\lambda Y$, we have $\widetilde{\mathscr{L}}\left(\mathscr{L}_{F, G}(Y)\right)=\theta\left(H_{n(m+1)}\right) \tilde{Y}=\lambda \tilde{Y}$, which yields $\sigma\left(\mathscr{L}_{F, G}\right)=\sigma\left(\theta\left(H_{n(m+1)}\right)\right)$. The above discussion concludes the proof of Theorem 4 . The proof of Theorem 4 is complete.

Remark 5. In Theorem 4, a necessary and sufficient condition for the asymptotically mean square stability of system (1) via the spectrum of $\mathscr{L}_{F, G}$ is presented, which can be called "spectral criterion."

Theorem 6. The trivial solution $x=0$ of system (1) is asymptotically mean square stable if and only if, for any $Q \in$ $S^{n(m+1)}$ with $Q>0$, there exists a $P \in S^{n(m+1)}$ such that $P>0$ and $P$ is a solution of the following Lyapunov equation:

$$
P-\mathscr{L}_{F, G}(P)=Q \text {. }
$$

Proof. We introduce an $n^{2}(m+1)^{2}$-parameter stochastic Lyapunov function as a quadratic form:

$$
V(\vec{X}(t))=\vec{X}^{\prime} P \vec{X}, \quad P \in S^{n(m+1)} .
$$

The role of parameters is played by $n^{2}(m+1)^{2}$ elements of the positive-definite matrix, which should be determined. The statement of the theorem can be established in a way that is standard for the method of Lyapunov functions for stochastic difference equations. So the trivial solution $\tilde{x}(t)=$ 0 of system (6) is asymptotically mean square stable if and only if for any $Q>0$, the Lyapunov equation (15) has a solution $P>0$. By the proof of Theorem 4 , the trivial solution $x=0$ of system (1) is asymptotically mean square stable if and only if the trivial solution $\tilde{x}(t)=0$ of system (6) is asymptotically mean square stable. The proof of Theorem 6 is complete.

From the proof of Theorem 4 and the method of Lyapunov functions for difference equations, we immediately get the following result.

Theorem 7. The trivial solution $x=0$ of system (1) is asymptotically mean square stable if and only if, for any $Q \in S^{n(m+1)[n(m+1)+1] / 2}$ with $Q>0$, there exists a $P \in$ $S^{n(m+1)[n(m+1)+1] / 2}$ such that $P>0$ and $P$ is a solution of the following Lyapunov equation:

$$
P-\widehat{F}^{\prime} P \widehat{F}=Q \text {. }
$$

Corollary 8. If $\sigma\left(\mathscr{L}_{F, G}\right) \subset D(0,1)$, then $\sigma(F) \subset D(0,1)$.

Proof. By Theorems 4 and $6, \sigma\left(\mathscr{L}_{F, G}\right) \subset D(0,1)$ holds if and only if there is a matrix $P \in S^{n(m+1)}$ such that $P>0$ and $P$ is a solution of the following Lyapunov equation:

$$
P-\mathscr{L}_{F, G}(P)=Q
$$

for any $Q>0$. So, there exists a $P \in P \in S^{n(m+1)}$ such that $P>0$ and $P$ is a solution of the following Lyapunov equation:

$$
P-F^{\prime} P F>0 \text {, }
$$

which is equivalent to $\sigma(F) \subset D(0,1)$; that is, the system

$$
\begin{gathered}
x(t+1)=F_{0} x(t)+\sum_{1}^{j=m} F_{j} x(t-j), \\
x(k)=\varphi(k), \quad k=0,-1,-2, \ldots,-m, t \in N,
\end{gathered}
$$

is asymptotically Lyapunov stable. The proof of Corollary 8 is complete.

Now, we present some results about mean square stability of system (1). From the process of Theorems 4-7, we easily obtain the following Theorems 9-10, so we omit their proofs.

Theorem 9. If the trivial stationary solution $x=0$ of the system (1) is mean square stable, then $\sigma\left(\mathscr{L}_{F, G}\right) \subset \bar{D}(0,1)$.

Theorem 10. $\sigma\left(\mathscr{L}_{F, G}\right) \subset \bar{D}(0,1)$ if and only if one of the following conditions holds.

(1) For any $\varepsilon>0$ and $Q>0$, the following Lyapunov equation

$$
P-\mathscr{L}_{e^{-\varepsilon} F, e^{-\varepsilon} G}(P)=Q
$$

has a positive-definite solution $P$.

(2) $\sigma\left(\mathscr{L}_{e^{-\varepsilon} F, e^{-\varepsilon} G}\right) \subset D(0,1)$.

Corollary 11. If $\sigma\left(\mathscr{L}_{F, G}\right) \subset \bar{D}(0,1)$, then $\sigma(F) \subset \bar{D}(0,1)$. 
Proof. Since $\sigma\left(\mathscr{L}_{F, G}\right) \subset \bar{D}(0,1)$, we have, for any sufficient small $\varepsilon>0, \sigma\left(\mathscr{L}_{e^{-\varepsilon} F, e^{-\varepsilon} G}\right) \subset D(0,1)$. By Theorem 4, $\left(e^{-\varepsilon} F, e^{-\varepsilon} G\right)$ is mean square stable, which implies $\sigma\left(\mathscr{L}_{e^{-\varepsilon} F}\right) \subset$ $D(0,1)$ by Corollary 8 . Let $\varepsilon \rightarrow 0$, we have $\sigma(F) \subset \bar{D}(0,1)$ by continuity of spectrum [16]. The proof of Corollary 11 is complete.

Remark 12. $\sigma(F) \subset \bar{D}(0,1)$ does not imply $\sigma\left(\mathscr{L}_{F, G}\right) \subset$ $\bar{D}(0,1)$, which is one of the essential differences between stochastic system and deterministic system.

Theorem 13. The trivial solution $x=0$ of system (1) is mean square stable if and only if, for any $Q \geq 0$, there exists a $P \in$ $S^{n(m+1)}$ such that $P>0$ and $P$ is a solution of the following Lyapunov equation:

$$
P-\mathscr{L}_{F, G}(P)=Q \text {. }
$$

Proof. We introduce an $n^{2}(m+1)^{2}$-parameter stochastic Lyapunov function as a quadratic form:

$$
V(\vec{X}(t))=\vec{X}^{\prime} P \vec{X}, \quad P \in R^{n(m+1) \times n(m+1)} .
$$

By the method of Lyapunov functions for stochastic difference equations, we can get the result. The proof of Theorem 13 is complete.

Now, we give an example to show how to solve the spectrum of stochastic time-delay deference system by $H$ representation.

Example 14. Consider the following stochastic system: $x(t+1)=a x(t)+b x(t-1)+c x(t) \omega(t)+d x(t-1) \omega(t)$.

Letting $\bar{x}(t)=(x(t), x(t-1))^{\prime}$,

$$
\bar{x}(t+1)=\left(\begin{array}{ll}
a & b \\
1 & 0
\end{array}\right) \bar{x}(t)+\left(\begin{array}{ll}
c & d \\
0 & 0
\end{array}\right) \bar{x}(t) \omega(t) .
$$

Letting $X(t)=E \bar{x}(t) \bar{x}^{\prime}(t)$,

$$
X(t+1)=\left(\begin{array}{ll}
a & b \\
1 & 0
\end{array}\right) X(t)\left(\begin{array}{ll}
a & 1 \\
b & 0
\end{array}\right)+\left(\begin{array}{ll}
c & d \\
0 & 0
\end{array}\right) X(t)\left(\begin{array}{ll}
c & 0 \\
d & 0
\end{array}\right) .
$$

Letting $F=\left(\begin{array}{cc}a & b \\ 1 & 0\end{array}\right), G=\left(\begin{array}{ll}c & d \\ 0 & 0\end{array}\right), \vec{X}(t)=\left(x_{11}, x_{12}, x_{12}, x_{22}\right)^{\prime}$,

$$
\begin{aligned}
\vec{X}(t+1) & =(F \otimes F+G \otimes G) \vec{X}(t) \\
& =\left(\begin{array}{cccc}
a^{2}+c^{2} & a b+c d & a b+c d & b^{2}+d^{2} \\
a & 0 & b & 0 \\
a & b & 0 & 0 \\
1 & 0 & 0 & 0
\end{array}\right) \vec{X}(t) .
\end{aligned}
$$

Choose

$$
H_{2}=\left(\begin{array}{lll}
1 & 0 & 0 \\
0 & 1 & 0 \\
0 & 1 & 0 \\
0 & 0 & 1
\end{array}\right)
$$

Let $\widetilde{X}(t)=\left(x_{11}, x_{12}, x_{22}\right)^{\prime}$; then $\vec{X}(t)=H_{2} \widetilde{X}(t)$ and

$$
\widetilde{X}(t+1)=\left(H_{2}^{\prime} H_{2}\right)^{-1} H_{2}^{\prime}(F \otimes F+G \otimes G) H_{2} \widetilde{X}(t) .
$$

So $\sigma\left(\left(H_{2}^{\prime} H_{2}\right)^{-1} H_{2}^{\prime}(F \otimes F+G \otimes G) H_{2}\right)=\sigma(\mathscr{L})$ if we choose $a=1, b=0, c=0, d=1 / 4$, and $\sigma(\mathscr{L})=\{0,1 / 2,1 / 2\}$.

For a state feedback control law $u(t)=K x(t)$, we introduce a linear operator $\mathscr{L}_{K}$ associated with the closedloop system:

$$
\begin{aligned}
& x(t+1)= F_{0} x(t)+M_{0} u(t)+\left(G_{0} x(t)+N_{0} u(t)\right) w(t) \\
&+\sum_{j=1}^{m}\left[F_{j} x(t-j)+M_{j} u(t-j)\right. \\
&\left.+\left(G_{j} x(t-j)+N_{j} u(t-j)\right) w(t)\right], \\
& x(k)=\varphi(k) \in R^{n}, \quad k=0,-1, \ldots,-m, t \in N,
\end{aligned}
$$

where $x \in R^{n}$ is a column vector, $F_{j}, G_{j} \in R^{n \times n}, j=0,1, \ldots, m$ are constant coefficient matrices, $\varphi(k)$ is a deterministic initial condition, and $u(t) \in R^{n}$ is a control input.

Definition 15. The trivial stationary solution $x(t)=0$ of the system (30) is called mean square stabilization if there exists an input feedback $K$ such that, for any arbitrarily small number $\varepsilon>0$, one can find a number $\delta>0$, when $\|\varphi\|<\delta$, satisfying

$$
E\|x(t)\|^{2}<\varepsilon
$$

for a solution $x(t)=x(t, \varphi)$ of $(30)$.

Definition 16. The trivial stationary solution $x(t)=0$ of the system (30) is called asymptotically mean square stabilization if it is stable in the sense of Definition 15 and, moreover,

$$
\lim _{t \rightarrow+\infty} E\|x(t)\|^{2}=0
$$

We introduce the following column vectors $\bar{x}(t)$ and $\bar{u}(t)$ of new variables of dimension $n(m+1)$ :

$$
\begin{aligned}
& \bar{x}(t)=\left[x^{\prime}(t), x^{\prime}(t-1), \ldots, x^{\prime}(t-m)\right]^{\prime}, \\
& \bar{u}(t)=\left[u^{\prime}(t), u^{\prime}(t-1), \ldots, u^{\prime}(t-m)\right]^{\prime} .
\end{aligned}
$$

The stochastic system (30) with time-delays can now be written in the form of an equivalent stochastic system of dimension $n(m+1)$ without time-delay; namely,

$$
\bar{x}(t+1)=\bar{F} \bar{x}(t)+\bar{M} \bar{u}(t)+(\bar{G} \bar{x}(t)+\bar{N} \bar{u}(t)) \omega(t),
$$


where

$$
\begin{aligned}
\bar{F} & =\left(\begin{array}{ccccc}
F_{0} & F_{1} & \cdots & F_{m-1} & F_{m} \\
I & 0 & \cdots & 0 & 0 \\
\vdots & \vdots & \cdots & \vdots & \vdots \\
0 & 0 & \cdots & I & 0
\end{array}\right), \\
\bar{G} & =\left(\begin{array}{ccccc}
G_{0} & G_{1} & \cdots & G_{m-1} & G_{m} \\
0 & 0 & \cdots & 0 & 0 \\
\vdots & \vdots & \vdots & \vdots & \vdots \\
0 & 0 & \cdots & 0 & 0
\end{array}\right) . \\
\bar{M} & =\left(\begin{array}{ccccc}
M_{0} & M_{1} & \cdots & M_{m-1} & M_{m} \\
0 & 0 & \cdots & 0 & 0 \\
\vdots & \vdots & \vdots & \vdots & \vdots \\
0 & 0 & \vdots & 0 & 0
\end{array}\right), \\
\bar{N} & =\left(\begin{array}{ccccc}
N_{0} & N_{1} & \cdots & N_{m-1} & N_{m} \\
0 & 0 & \cdots & 0 & 0 \\
\vdots & \vdots & \vdots & \vdots & \vdots \\
0 & 0 & \cdots & 0 & 0
\end{array}\right) .
\end{aligned}
$$

Take a control input $\bar{u}(t)=\bar{K} \bar{x}(t)$ with

$$
\bar{K}=\left(\begin{array}{ccccc}
K & 0 & \cdots & 0 & 0 \\
0 & K & \cdots & 0 & 0 \\
\vdots & \vdots & \vdots & \vdots & \vdots \\
0 & 0 & \cdots & 0 & K
\end{array}\right),
$$

and set $X(t)=E \bar{x}(t) \bar{x}^{\prime}(t) ; X(t)$ satisfies the following difference equation:

$$
\begin{aligned}
X(t+1)= & (\bar{F}+\overline{M K}) X(t)(\bar{F}+\overline{M K})^{\prime} \\
& +(\bar{G}+\overline{N K}) X(t)(\bar{G}+\overline{N K})^{\prime} .
\end{aligned}
$$

Motivated by (38), we introduce the following linear Lyapunov operator:

$$
\begin{aligned}
\mathscr{L}_{\bar{K}}: X \in S^{n(m+1)} \longmapsto(\bar{F}+\overline{M K}) X(t)(\bar{F}+\overline{M K})^{\prime} \\
+(\bar{G}+\overline{N K}) X(t)(\bar{G}+\overline{N K})^{\prime} \in S^{n(m+1)} .
\end{aligned}
$$

With the use of the Kronecker matrix product, the matrix equation (38) can be rewritten in the vector matrix form as follows:

$$
\vec{X}(t+1)=\widehat{A} \vec{X},
$$

where $\vec{X}(t)$ denotes the $n^{2}(m+1)^{2}$-dimensional column vector

$$
\begin{aligned}
\vec{X}(t)= & {\left[X_{1,1}(t), \ldots, X_{1, n}(t), \ldots,\right.} \\
& \left.X_{1, n(m+1)}(t), \ldots, X_{n(m+1), n(m+1)}(t)\right]^{\prime}
\end{aligned}
$$

and $\widehat{A}=(\bar{F}+\overline{M K}) \otimes(\bar{F}+\overline{M K})+(\bar{G}+\overline{N K}) \otimes(\bar{G}+\overline{N K}) \epsilon$ $S^{n^{2}(m+1)^{2}}$.

From Theorems 4-13, we can easily obtain the following results.

Corollary 17. The trivial solution $x=0$ of system (30) is asymptotical mean square stabilizaton if and only if $\sigma\left(\mathscr{L}_{\bar{K}}\right) \subset$ $D(0,1)$.

Corollary 18. The trivial solution $x(t)=0$ of system (30) is asymptotically mean square stable if and only if, for any $Q \in$ $S^{n(m+1)}$ with $Q>0$, there exists a $P \in S^{n(m+1)}$ such that $P>0$ and $P$ is a solution of the following Lyapunov equation:

$$
P-\mathscr{L}_{\bar{F}+\overline{M K}, \bar{G}+\overline{N K}}(P)=Q .
$$

Corollary 19. The trivial solution $x=0$ of system (30) is asymptotically mean square stable if and only if, for any $Q \in S^{n(m+1)[n(m+1)+1] / 2}$ with $Q>0$, there exists a $P \in$ $S^{n(m+1)[n(m+1)+1] / 2}$ such that $P>0$ and $P$ is a solution of the following Lyapunov equation:

$$
P-\theta\left(H_{n(m+1)}^{K}\right)^{\prime} P \theta\left(H_{n(m+1)}^{K}\right)=Q,
$$

where

$$
\begin{aligned}
\theta\left(H_{n(m+1)}^{K}\right)=[ & \left.H_{n(m+1)}^{\prime} H_{n(m+1)}\right]^{-1} H_{n(m+1)}^{\prime} \\
\times & {[(\bar{F}+\overline{M K}) \otimes(\bar{F}+\overline{M K})} \\
& +(\bar{G}+\overline{N K}) \otimes(\bar{G}+\overline{N K})] H_{n(m+1)} .
\end{aligned}
$$

Corollary 20. The trivial solution $x=0$ of system (30) is asymptotically mean square stable if and only if, for any $Q \in$ $S^{n^{2}(m+1)^{2}}, Q>0$, there exists a $P \in S^{n^{2}(m+1)^{2}}$ such that $P>0$ and $P$ is a solution of the following Lyapunov equation:

$$
P-\widehat{A}^{\prime} P \widehat{A}=Q .
$$

Corollary 21. The trivial solution $x=0$ of system (30) is mean square stable if and only if, for any $Q \in S^{n(m+1)}$ with $Q \geq 0$, there exists a $P \in S^{n(m+1)}$ such that $P>0$ and $P$ is a solution of the following Lyapunov equation:

$$
P-\mathscr{L}_{\bar{F}+\overline{M K}, \bar{G}+\overline{N K}}(P)=Q .
$$

Corollary 22. The trivial solution $x=0$ of system (1) is mean square stable if and only if, for any $Q \in S^{n(m+1)[n(m+1)+1] / 2}$ with $Q \geq 0$, there exists a $P \in S^{n(m+1)[n(m+1)+1] / 2}$ such that $P>0$ and $P$ is a solution of the following Lyapunov equation:

$$
P-\theta\left(H_{n(m+1)}^{K}\right)^{\prime} P \theta\left(H_{n(m+1)}^{K}\right)=Q,
$$

where

$$
\begin{aligned}
\theta\left(H_{n(m+1)}^{K}\right)= & {\left[H_{n(m+1)}^{\prime} H_{n(m+1)}\right]^{-1} } \\
& \times H_{n(m+1)}^{\prime}[F \otimes F+G \otimes G] H_{n(m+1)} .
\end{aligned}
$$


Corollary 23. The trivial solution $x=0$ of system (1) is mean square stable if and only if, for any $Q \geq 0$, there exists a $P \in$ $S^{n^{2}(m+1)^{2}}$ such that $P>0$ and $P$ is a solution of the following Lyapunov equation:

$$
P-\widehat{A}^{\prime} P \widehat{A}=Q
$$

\section{Popov-Belevith-Hautus Criterion of the Stabilizability}

In this section, we will investigate the properties of unremovable spectrum of time-delay deference system and the relation between unremovable spectrum and the stabilizability of time-delay deference system. Consider the following linear stochastic system with time-delays:

$$
\begin{array}{r}
x(t+1)=F_{0} x(t)+M_{0} u(t)+\left(G_{0} x(t)+N_{0} u(t)\right) w(t) \\
+\sum_{j=1}^{m}\left[F_{j} x(t-j)+M_{j} u(t-j)\right. \\
\left.\quad+\left(G_{j} x(t-j)+N_{j} u(t-j)\right) w(t)\right], \\
x(k)=\varphi(k) \in R^{n}, \quad k=0,-1,-2, \ldots,-m, t \in N .
\end{array}
$$

Definition 24. We say that $\lambda$ is an unremovable spectrum of system (50) with state feedback if there exists $Z \neq 0 \in S^{n(m+1)}$, such that,for any $\bar{K} \in R^{n(m+1) \times n(m+1)}$,

$$
\begin{aligned}
\mathscr{L}_{\bar{K}}^{*}(Z)= & (\bar{F}+\overline{M K})^{\prime} Z(\bar{F}+\overline{M K}) \\
& +(\bar{G}+\overline{N K})^{\prime} Z(\bar{G}+\overline{N K})=\lambda Z
\end{aligned}
$$

holds.

Remark 25. It is easy to see that the operator $\mathscr{L}_{\bar{K}}^{*}$ is the adjoint operator of the operator $\mathscr{L}_{\bar{K}}$ with the inner product $\langle Z, Y\rangle=$ $\operatorname{trace}\left(Z^{*}, Y\right)$ for any $Z, Y \in S^{n(m+1)}$. As we restrict the coefficients to real matrices, $\sigma\left(\mathscr{L}_{\bar{K}}^{*}\right)=\sigma\left(\mathscr{L}_{\bar{K}}\right)$. By Corollaries $17-23$, we know that any one of them can characterize the stabilizability of system (50).

Obviously, if $\lambda$ is an unremovable spectrum, then it can be regarded as an uncontrollable mode as in deterministic systems. We give a theorem with respect to the unremovable spectrum below.

Theorem 26. (Stochastic PBH criterion) $\lambda$ is an unremovable spectrum of system (50) if and only if there exists $Z \neq 0 \in$ $S^{n(m+1)}$, such that the following three equalities hold simultaneously:

$$
\begin{aligned}
& \bar{F}^{\prime} Z \bar{F}+\bar{G}^{\prime} Z \bar{G}=\lambda Z, \\
& \bar{F}^{\prime} Z \bar{M}+\bar{G}^{\prime} Z \bar{N}=0, \\
& \bar{N}^{\prime} Z \bar{N}=-\bar{M}^{\prime} Z \bar{M} .
\end{aligned}
$$

Proof. Note that (51) can be written as

$$
\begin{aligned}
& \bar{F}^{\prime} Z \bar{F}+\bar{K}^{\prime} \bar{M}^{\prime} Z \overline{M K}+\bar{G}^{\prime} Z \bar{G}^{\prime}+\bar{K}^{\prime} \bar{N}^{\prime} Z \overline{N K} \\
& +\left(\bar{F}^{\prime} Z \bar{M}+\bar{G}^{\prime} Z \bar{N}\right) \bar{K}+\bar{K}^{\prime}\left(\bar{M}^{\prime} Z \bar{F}+\bar{N}^{\prime} Z \bar{G}\right)=\lambda Z,
\end{aligned}
$$

so if (52) holds, then (53) automatically holds. So the sufficiency is proved.

To prove the necessity, we first take $\bar{K}=0$ in (51), then

$$
\bar{F}^{\prime} Z \bar{F}+\bar{G} Z \bar{G}^{\prime}=\lambda Z
$$

holds. Again, from (53), it follows that

$$
\begin{aligned}
& \bar{K}^{\prime} \bar{M} Z \bar{M}^{\prime} \bar{K}+\overline{K N} Z \bar{N}^{\prime} \bar{K}^{\prime} \\
& \quad+\left(\bar{F}^{\prime} Z \bar{M}+\bar{G}^{\prime} Z \bar{N}\right) \bar{K}+\bar{K}^{\prime}\left(\bar{M}^{\prime} Z \bar{F}+\bar{N}^{\prime} Z \bar{G}\right)=0 .
\end{aligned}
$$

Let $\mathscr{F}=\bar{F}^{\prime} Z \bar{M}+\bar{G}^{\prime} Z \bar{N}, \mathscr{M}=\bar{M} Z \bar{M}^{\prime}, \mathcal{N}=\bar{N} Z \bar{N}^{\prime}$; then (55) becomes

$$
\mathscr{F} \bar{K}+\bar{K}^{\prime} \mathscr{F}^{\prime}=-\bar{K}^{\prime} \mathscr{M} \bar{K}-\bar{K}^{\prime} \mathscr{N} \bar{K} .
$$

Since the left-hand side in (56) is linear with respect to $\bar{K}$, we must have $\mathscr{M}+\mathscr{N}=0$. In fact, due to the linearity of the following equation

$$
\begin{aligned}
(\bar{K} & +\bar{K})^{\prime}(\mathscr{M}+\mathcal{N})(\bar{K}+\bar{K}) \\
& =4 \bar{K}^{\prime}(\mathscr{M}+\mathscr{N}) \bar{K}=2 \bar{K}^{\prime}(\mathscr{U}+\mathscr{N}) \bar{K}
\end{aligned}
$$

$\bar{K}^{\prime}(\mathscr{M}+\mathcal{N}) \bar{K}=0$. Because of the arbitrariness of $\bar{K}$, it is necessary that $\mathscr{M}+\mathcal{N}=0$; that is, $\bar{M} Z \bar{M}^{\prime}=-\bar{N} Z \bar{N}^{\prime}$. To prove $\mathscr{F}=0$ or $\bar{F}^{\prime} Z \bar{M}+\bar{G}^{\prime} Z \bar{N}=0$, we note that (56) becomes $\bar{K}^{\prime} \mathscr{F}^{\prime}=-\mathscr{F} \bar{K}$. Denote $\mathscr{F}=\left(f_{i j}\right)_{n(m+1) \times n(m+1)}$, and take

$$
\bar{K}=\bar{K}_{i j}=\left(k_{l s}\right)_{n(m+1) \times n(m+1)}= \begin{cases}1, & l=i, s=j, \\ 0, & \text { otherwise }\end{cases}
$$

From $\bar{K}^{\prime} \mathscr{F}^{\prime}=-\mathscr{F} \bar{K}$, one knows that $f_{i j}=0, i, j=$ $1,2, \ldots, n(m+1)$; that is, $\mathscr{F}=0$. The proof of Theorem 26 is complete.

Theorem 27. If system (50) is asymptotically mean square stabilizable, then all unremovable spectra of system (50) must belong to $D(0,1)$.

Proof. If there is an unremovable spectrum $\mu$ of (50) with $|\mu| \geq 1$, then, by Theorem 26 , there exists $Z \neq 0 \in S^{n(m+1)}$, such that the following three equalities hold simultaneously:

$$
\begin{aligned}
& \bar{F}^{\prime} Z \bar{F}+\bar{G}^{\prime} Z \bar{G}=\mu Z, \\
& \bar{F}^{\prime} Z \bar{M}+\bar{G}^{\prime} Z \bar{N}=0, \\
& \bar{N}^{\prime} Z \bar{N}=-\bar{M}^{\prime} Z \bar{M} .
\end{aligned}
$$


So, for any feedback gain $\bar{K}$, we obtain $\mathscr{L}_{\bar{K}}(Z)=\mu Z, \mu \notin$ $D(0,1)$. The proof of Theorem 27 is complete. It follows that, for any $\bar{K}, \mu \in \sigma\left(\mathscr{L}_{\bar{K}}\right)$, which contradicts the asymptotical mean square stabilization of system (50).

By Theorems 26 and 27, the deterministic PopovBelevith-Hautus Criterion can be stated in another form as follows.

Corollary 28. Assume that $G_{j}=N_{j}=0, j=0,1, \ldots, m$; then system (50) is asymptotically stabilizable if and only if all of the unremovable spectra of system (50) belong to $D(0,1)$; that is, there does not exist a nonzero $Z \in S^{n(m+1)}$, and $\lambda \notin D(0,1)$ satisfying

$$
\bar{F}^{\prime} Z \bar{F}=\lambda Z, \quad Z \bar{M}=0 .
$$

Proof. The necessity is obvious. To prove the sufficiency part, we note that if $\left(F_{0}, M_{0}\right)$ is not stabilizable, then, by PopovBelevith-Hautus Criterion, there exists a nonzero $\xi \in C^{n(m+1)}$, $\lambda \notin D(0,1)$ satisfying $\xi^{\prime} \bar{F}=\lambda \xi^{\prime}, \xi^{\prime} \bar{M}=0$. Take $Z=$ $\xi \xi^{\prime}$, then (60) holds, which contradicts the given condition. Corollary 28 is proved.

Remark 29. Corollary 28 indicates that there is no difference between unremovable spectrum and uncontrollable mode for deterministic systems.

Theorem 30. If system (50) is mean square stabilizable, then all the existing unremovable spectra of (50) must belong to $\bar{D}(0,1)$.

Proof. If there is an unremovable spectrum $\mu$ of system (50) with $\mu \notin \bar{D}(0,1)$, then, by Theorem 26 , there exists $Z \neq 0 \in S^{n(m+1)}$, such that the following three equalities hold simultaneously:

$$
\begin{aligned}
& \bar{F}^{\prime} Z \bar{F}+\bar{G}^{\prime} Z \bar{G}=\mu Z, \\
& \bar{F}^{\prime} Z \bar{M}+\bar{G}^{\prime} Z \bar{N}=0, \\
& \bar{N}^{\prime} Z \bar{N}=-\bar{M}^{\prime} Z \bar{M} .
\end{aligned}
$$

So for any feedback gain $\bar{K}$, we obtain $\mathscr{L}_{\bar{K}}^{*}(Z)=\mu Z$, $\mu \notin \bar{D}(0,1)$. It follows that, for any $\bar{K}, \mu \in \sigma\left(\mathscr{L}_{\bar{K}}\right)$, which contradicts mean square stabilization of system (50). The proof of Theorem 30 is complete.

\section{Conclusion}

In this paper, we investigate the stability and stabilizability of stochastic delay-time systems. By $H$-representation, present the spectral criteria of the stability and stabilizability. By generalized Lyapunov equation approach, the equivalent conditions of mean square stabilizability and asymptotically mean square stabilizability of system (50) are given. We introduce the notion of unremovable spectrum of stochastic time-delay deference system, present the $\mathrm{PBH}$ criterion of the unremovable spectrum of stochastic time-delay system, and investigate the relation between the unremovable spectrum and the stabilizability of stochastic time-delay deference system.

\section{Conflict of Interests}

The authors declare that there is no conflict of interests regarding the publication of this paper.

\section{Acknowledgment}

This work was supported by the National Natural Science Foundation of China under Grant nos. 61174078 and 61201430

\section{References}

[1] A. G. Mazko, "The Lyapunov matrix equation for a certain class of regions bounded by algebraic curves," Soviet Automatic Control, vol. 42, pp. 12-17, 1980.

[2] R. Z. Has'minskii, Stochastic Stability of Differential Equations, vol. 7 of Monographs and Textbooks on Mechanics of Solids and Fluids: Mechanics and Analysis, Sijtjoff and Noordhoff, Alphen, The Netherlands, 1980.

[3] H. J. Kushner, Stochastic Stability and Control, vol. 33 of Mathematics in Science and Engineering, Academic Press, New York, NY, USA, 1967.

[4] M. A. Rami and X. Y. Zhou, "Linear matrix inequalities, Riccati equations, and indefinite stochastic linear quadratic controls," IEEE Transactions on Automatic Control, vol. 45, no. 6, pp. 1131$1143,2000$.

[5] T. Damm, Rational Matrix Equations in Stochastic Control, vol. 297 of Lecture Notes in Control and Information Sciences, Springer, New York, NY, USA, 2004.

[6] X. Mao, Stochastic Differential Equations and Their Applications, Horwood, Chichester, UK, 1997.

[7] Y. Liu, Backward stochastic differential equation and stochastic control system [Ph.D. dissertation], Shandong University, Jinan, China, 1999.

[8] S. Boyd, L. El Ghaoui, E. Feron, and V. Balakrishnan, Linear Matrix Inequalities in System and Control Theory, vol. 15, SIAM, Philadelphia, Pa, USA, 1994.

[9] M. D. Fragoso, O. L. V. Costa, and C. E. de Souza, "A new approach to linearly perturbed Riccati equations arising in stochastic control," Applied Mathematics and Optimization, vol. 37, no. 1, pp. 99-126, 1998.

[10] W. M. Wonham, "On a matrix Riccati equation of stochastic control," SIAM Journal on Control, vol. 6, no. 4, pp. 681-697, 1968.

[11] A. Pazy, Semigroups of Linear Operators and Applications to Partial Differential Equations, vol. 44 of Applied Mathematical Sciences, Springer, New York, NY, USA, 1983.

[12] W. Zhang, H. Zhang, and B.-S. Chen, "Generalized Lyapunov equation approach to state-dependent stochastic stabilization/detectability criterion," IEEE Transactions on Automatic Control, vol. 53, no. 7, pp. 1630-1642, 2008.

[13] W. Zhang and B. S. Chen, "Some properties of generalized Lyapunov equations," in Proceedings of the Chinese Control and Decision Conference (CCDC '11), pp. 3137-3141, Mianyang, China, May 2011. 
[14] W. Zhang and B. S. Chen, " $H$-representation and applications to generalized Lyapunov equations and linear stochastic systems," IEEE Transactions on Automatic Control, vol. 57, no. 12, pp. 3009-3022, 2012.

[15] W. Zhang and B. S. Chen, "On stabilizability and exact observability of stochastic systems with their applications," Automatica, vol. 40, no. 1, pp. 87-94, 2004.

[16] D. G. Korenevskii, "Stability criteria for solutions of systems of linear deterministic or stochastic delay difference equations with continuous time," Mathematical Notes, vol. 70, no. 1-2, pp. 192-205, 2001. 


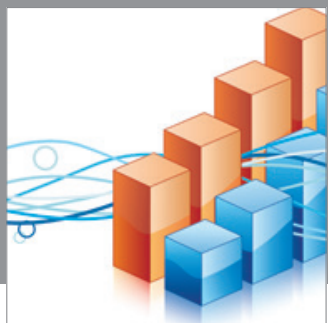

Advances in

Operations Research

mansans

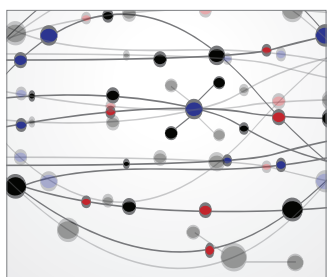

The Scientific World Journal
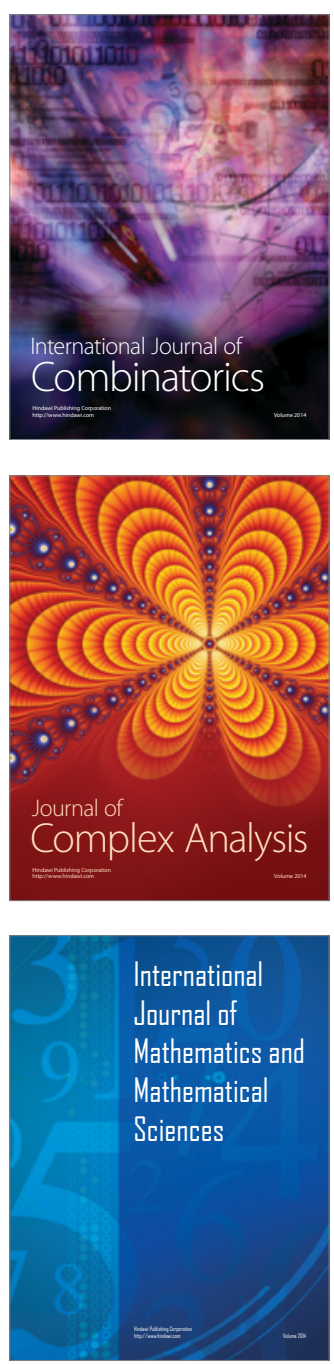
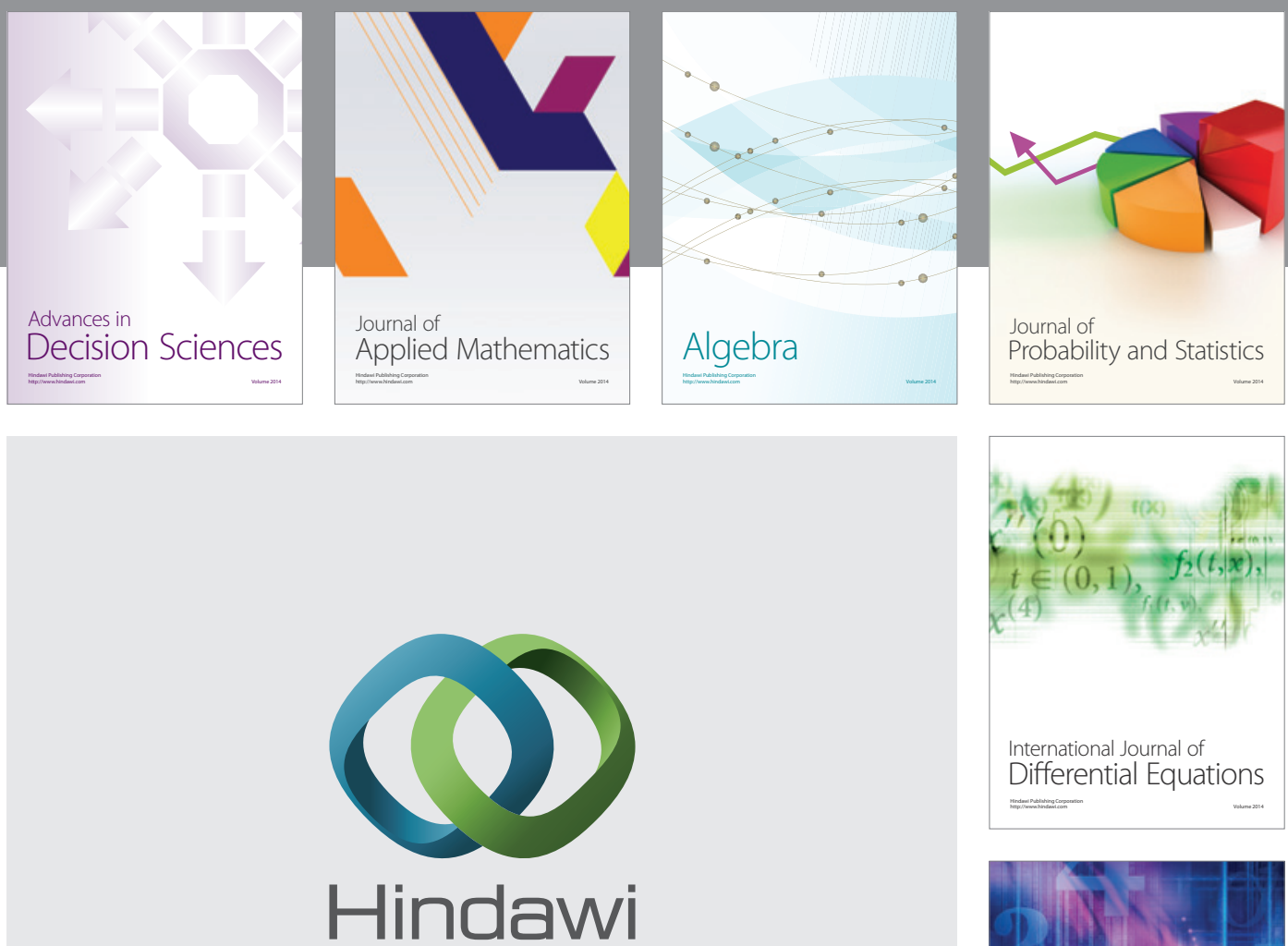

Submit your manuscripts at http://www.hindawi.com
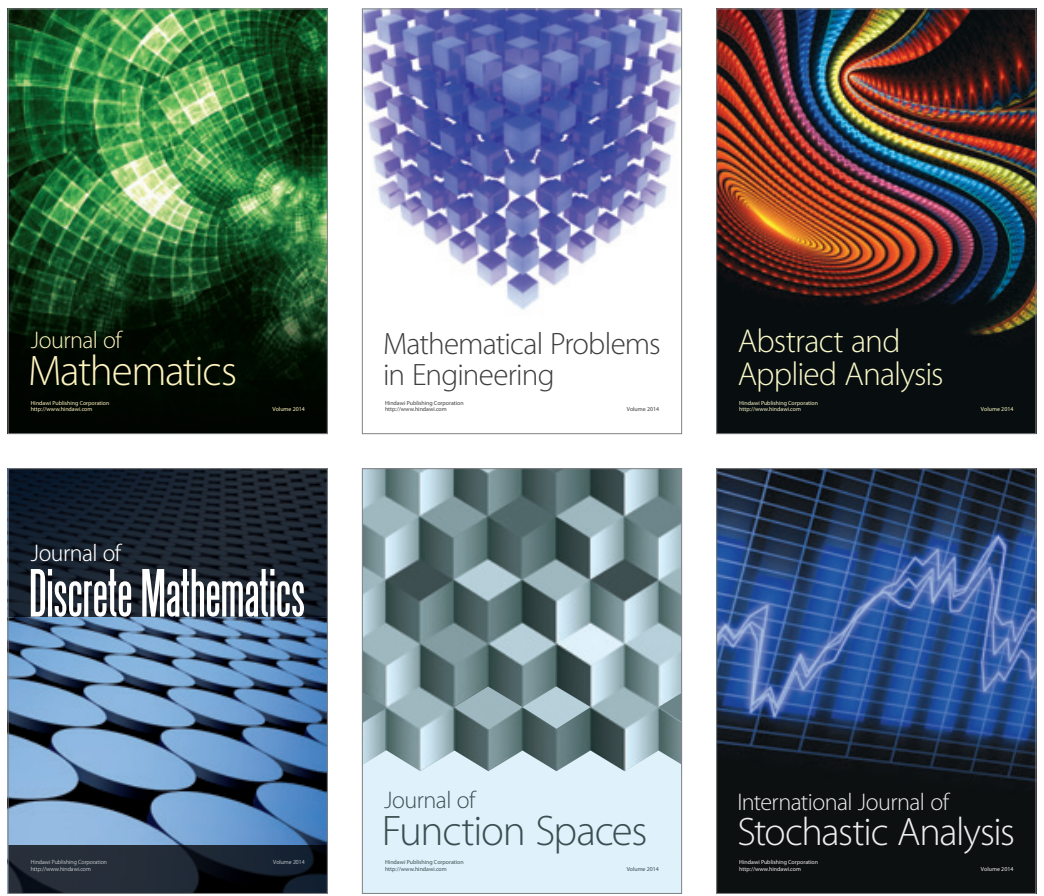

Journal of

Function Spaces

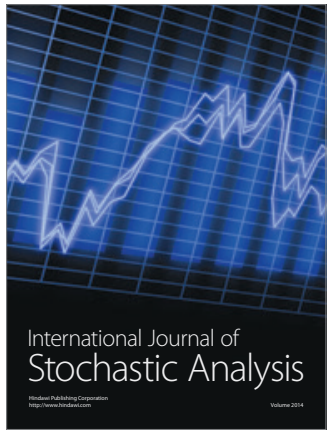

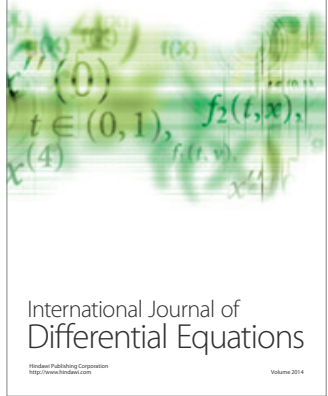
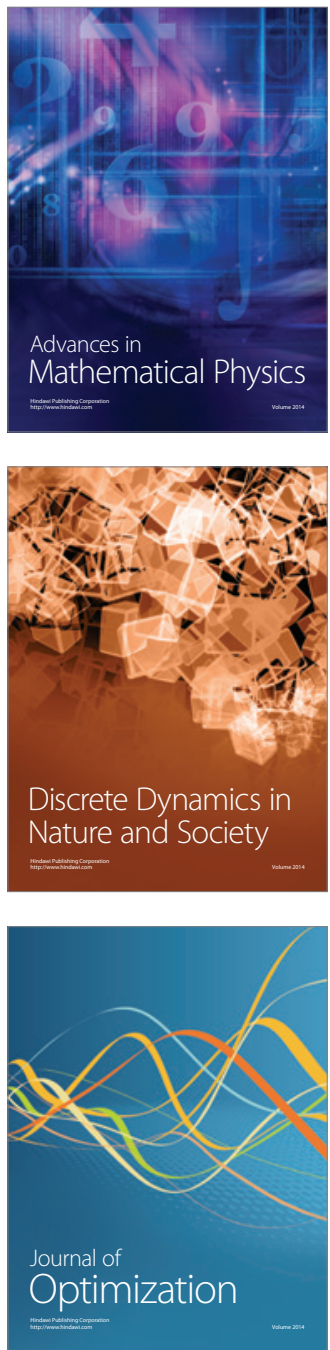